\title{
Commencement de parti
}

\author{
Shelley Stern et Anne Wagner
}

\section{Décor}

Grand bureau typiquement bureaucrate. Sur les deux murs, une douzaine de portraits identiques, sur lesquels figure un homme en lunettes et cravate, avec une moustache qui ne peut cacher son sourire épanoui. La partie basse des murs est recouverte d'étagères ou se trouvent 300 exemplaires du même livre intitulé Comment Faire 1a Cuisine.

Nous avons essayé de Zaisser le metteur-enscène libre d'interpréter notre pièce comme il l'entend; tout ce que nous demandons, c'est qu'il nous envoie des biliets à titre gracieux pour la première représentation.

On remarquera le nom des personnages. "QueZqu'un" veut dire exactement cela; quelqu'un doit donner la réplique, mais peu importe qui. Remarquer la chanson "Révolution" par les Beatles. Elze s'applique à cette piece, aussi bien que la chanson "Révolution $n^{\circ} g$ "; nous suggérons la dernière comme prélude, si l'on veut.

\section{Scène I}

(Les personnages entrent, à Zoisir.) 
On a gagné la révolution.

$$
\text { QUELQU'UN }
$$

QUELQU'UN

$\mathrm{Qu}$ 'est-ce qu'on fait maintenant?

QUELQU'UN

Ça nous a pris vingt ans.

$$
\text { UNE VOIX }
$$

Et qu'est-ce qu'on fait maintenant? QUELQU'UN

Où puis-je acheter un hamburger?

(Sizence.)

$$
\text { QUELQU'UN }
$$

Il n'y a plus de viande depuis hier. TOUS

Nous en sommes très fiers...

On a tout fait sauter en $1^{\prime}$ air!

QUELQU'UN

Les jardins, les jardinières.

$$
\text { QUELQU'UN }
$$

Les soeurs, les frères.

$$
\text { QUELQU'UN }
$$

Les soutien-gorge, les jarretières. QUELQU'UN

Les cuisines, les cuisinières. QUELQU' 'UN

Il n'y a plus de viande depuis hier. TOUS

Mais, nous, nous pouvons tout faire! QUELQU 'UN

C'est ça, on trouvera une substitution. QUELQU'UN

Commençant par les institutions... QUELQU'UN

Les écoles-maternelles? QUELQU 'UN

Les bureaux, leur personnel? 


\section{QUELQU'UN}

Les petits enfants tendres et croustillants...

UNE VOIX

Et qu'est-ce qu'on va faire maintenant?

DE LA VIANDE! TOUS

Comment?

$$
\text { QUELQU'UN }
$$

Hachée?

$$
\text { QUELQU'UN }
$$

Escalopée?

$$
\text { QUELQU'UN }
$$

Rọuladée?

$$
\text { QUELQU'UN }
$$

Enveloppée?

$$
\text { QUELQU'UN }
$$

MARINETTE

Pardon, Monsieur, avez-vous de la moutarde? MARINOT

Non, Mademoiselle, nous ne servons que de la sauce hollandaise.

QUELQU'UN

Ga nous a pris deux cents ans. QUELQU'UN

Ça nous prendra une éternité. QUELQU 'UN

Le pouvoir est à nous dorénavant. QUELQU'UN

Remarquez que le monde est dépeuplé.

(Sizence.)

$$
\text { MARINOT, déclamatoire }
$$

Buvez! Il faut boire à toutes les sources.

Chacun son propre menu.

$\mathrm{Ne}$ vous inquiétez pas; nos bourses

Sont toujours bien pourvues.

(Ils tournent tous le dos à l'auditoire.) 


\section{Scène II}

\section{PORTE-PAROLE}

Maître Président, sur les murs imprimés, Tient sur sa bouche un sourire. Maître LePeuple, par le pouvoir alléché, Le regarde, en commençant à dire.

(Tous se remettent en face de l'auditoire.)

o! bonjour, Monsieur le Président!

Comme vous êtes joli!

$$
\text { QUELQU'UN }
$$

$$
\text { QUELQU'UN }
$$

Comme vous nous semblez beau!

$$
\text { QUELQU'UN }
$$

Sans doute, si votre sourire

Se rapporte à votre plaisir,

$$
\text { TOUS }
$$

Vous êtes le plus drôle des révolutionnaires.

$$
\text { MARINOT }
$$

Je trouve qu'il est bien mignon, n'est-ce pas, Marinette?

\section{MARINETTE}

Son petit air de vieux fou, Avec cravate et lunettes...

MARINOT

Il n'est pas parmi nous...

$$
\text { MARINETTE }
$$

Mais si, mon ami,

I1 nous observe,

I1 nous voit.

C'est notre grand-frère,

$C^{\prime}$ 'est notre prédécesseur,

$C^{\prime}$ 'est notre successeur,

$C^{\prime}$ est notre pasteur.

MARINOT

Mais puisque nous 1 'avons mis à mort... 


\section{MARINETTE, pas troublée}

Oui, 1a révolution doit tout purger,

Tout exterminer...

\section{TOUS}

Il est crevé

COMME UNE CREVETTE

Crustacée.

Donc, puisqu'il n'y a plus

QUELQU'UN

De viande...

TOUS

Nous mangerons les poissons. QUELQU'UN

Mais 1 'océan s'est évaporé. QUELQU'UN

La Révolution, nous la faisons. QUELQU'UN

Le Mal, c'est le passé.

TOUS

Le Parti a toujours raison,

Il a fallu le rejeter.

$$
\text { UNE VOIX }
$$

Et qu'est-ce qu'on fait maintenant?

$$
\text { TOUS }
$$

On chante le cantique de la révolution:

L'Idée qui répand la terreur,

L'Idée que le ciel dans sa fureur

Octroya pour punir les capitalistes de la terre, Le Parti (puisqu'il faut 1'appeler par son nom)

Donna naissance aux révolutionnaires.

(C'est nous! C'est nous!)

Ils ne mouraient pas tous,

Mais tous étaient frappés.

On ne voyait point d'inoccupés, Chaque mets excitait notre envie.

(Tous se fézicitent, bien contents de leur parti, ainsi que de leur succès.) 


\section{MARINETTE}

J'ai encore besoin de moutarde.

TOUS

Elle en a besoin.

MARINOT

Eh bien, Mademoiselle, il faut vous satisfaire...

(Parlant comme un commis-voyageur.)

Euh... Ah oui, ce drapeau, vous plâ̂t-il?

(Comme iz se dresse dans le vent.)

Ne voudriez-vous pas le manger? Voici un couteau.

\section{MARINETTE}

Si vous me souriez

Je le ferai, je le tiendrai.

(Quelqu'un donne une copie du Zivre de cuisine à Marinette. Elle prend le livre et le couteau en même temps et commence à lire le livre et $\grave{a}$ manger le dropeau à la fois. Ici le metteur-enscène peut montrer son ingéniosité et son originalité par sa représentation.)

TOUS

La Révolution nous mange.

(On entend "Volunteers" par Le Jefferson Airplane.)

UNE VOIX

Et qu'est-ce qu'on fait maintenant?

QUELQU'UN

Ça nous a pris beaucoup de temps.

QUELQU 'UN

Ça finira dans peu de temps.

J'entends 1 'aube. 
Elle ne vient pas?

$$
\text { QUELQU' 'UN }
$$

E1le ne dit rien.

$$
\text { QUELQU'UN }
$$

$$
\text { QUELQU'UN }
$$

I1 faut entendre les promesses du Parti.

$$
\text { QUELQU'UN }
$$

Je brûle,

Il a été brûlé.

Quelque chose brûle.

$$
\text { TOUS }
$$

MARINETTE

La viande, on 1'a trop brûlée.

(Elle mange le drapeau.)

Il faut le recouvrir de sauce hollandaise. UNE VOIX

La nuit doit venir,

I1 faut la saisir, la tenir

Dans ses mains avant l'aube.

(Tous tournent le dos à l'auditoire.)

\section{Scène III}

(On entend le cri d'un enfant. Tous se remettent en face de l'auditoire. Maintenant ils sont tous des enfants. Personne n'a plus de sept ans.)

On a gagné le jeu.

$$
\text { QUELQU' UN }
$$

$$
\text { QUELQU'UN, doucement }
$$

Je veux encore y jouer un petit peu.

$$
\text { QUELQU' UN }
$$

Ça fait déjà deux minutes.

Allons ailleurs.

QUELQU'UN 


\section{QUELQU' UN}

Et qu'est-ce qu'on fait maintenant?

\section{PETIT GARCON}

Jouons à quelque chose...

Jouons au soldat!

$$
\text { QUELQU'UN }
$$

L'Etat, ce sera moi!

$$
\text { PETIT GARCON }
$$

Avec vous tous comme marionnettes.

Je jugerai ce que vous faites;

C'est le temps de voir ce que vous vaut.

Valez!

$$
\text { PORTE-PAROLE, à part }
$$

\section{PETIT PRINCE, férocement}

Quoi? Vous NOUS interrompez? Idiot! Imbécile! Salaud!

(Le Porte-Parole mange ses cheveux.)

$$
\text { PORTE-PAROLE, haletant, sanglotant }
$$

Mes yeux doivent se fermer,

Je ne vois qu'un néant...

En bouillonnant il me fait brûler;

Ca n'a pris que peu de temps

J'entre dans le feu...

Adieu... mes amis... à... Dieu.

(Le Porte-Parole ferme les yeux et quitte la scène.)

\section{PETIT PRINCE}

Nous sommes à notre affaire;

Nous pouvons commencer.

Je réclame ma mère!

$$
\text { QUELQU'UN }
$$

$$
\text { QUELQU'UN }
$$

Pour qu'elle me donne un petit frère.

$$
\text { PETIT PRINCE }
$$

Nous vous ordonnons de vous taire!

C'est moi qui suis le roi, 
C'est moi qui fais la loi.

MARIONNETTES

C'est toi qui es le roi,

C'est toi qui fais la loi.

PETIT PRINCE, pas encore satisfait

Je veux régner entièrement dans 1'air.

MARINETTE, les joues rouges, comme une poupée

Mais... il faut... d'abord...

(Comme si elle avait un grand secret)

la guerre!!!

(Joyeusement, elle frappe des mains.)

C'est ça! c'est ça!

MARIONNETTES

II nous faut une guerre

Grande et belle.

MARIONNETTE

Bella, bellae, bellum!

La Belle au Bois Dormant!

La Belle et la Bête!

TOUS

C'est ça! La guerre!

La Belle est la Bête!

MARINETTE

Maman m'a dit qu'aujourd'hui

Avec la paix les gens s'ennuient.

UNE VOIX

Et qu'est-ce qu'on fait maintenant?

$$
\text { TOUS }
$$

On se débarrasse des parents.

On se débarrasse des tourments.

UNE VOIX

La révolte ne saurait jouer longtemps le rôle de la Révolution.

\section{PETIT PRINCE}

Eh bien. Continuons à commencer. 
(Tous se groupent dans un cercle. Ils commencent d jouer aux "Catégories," un jeu d'enfants.)

\section{ENVOI}

Si vous pensez que c'est un jeu,

Ça va...

Faites-en ce que vous voulez.

Nous nous sommes présentés

Maintenant, c'est à vous de décider.

Oakland University

Rochester, Michigan 


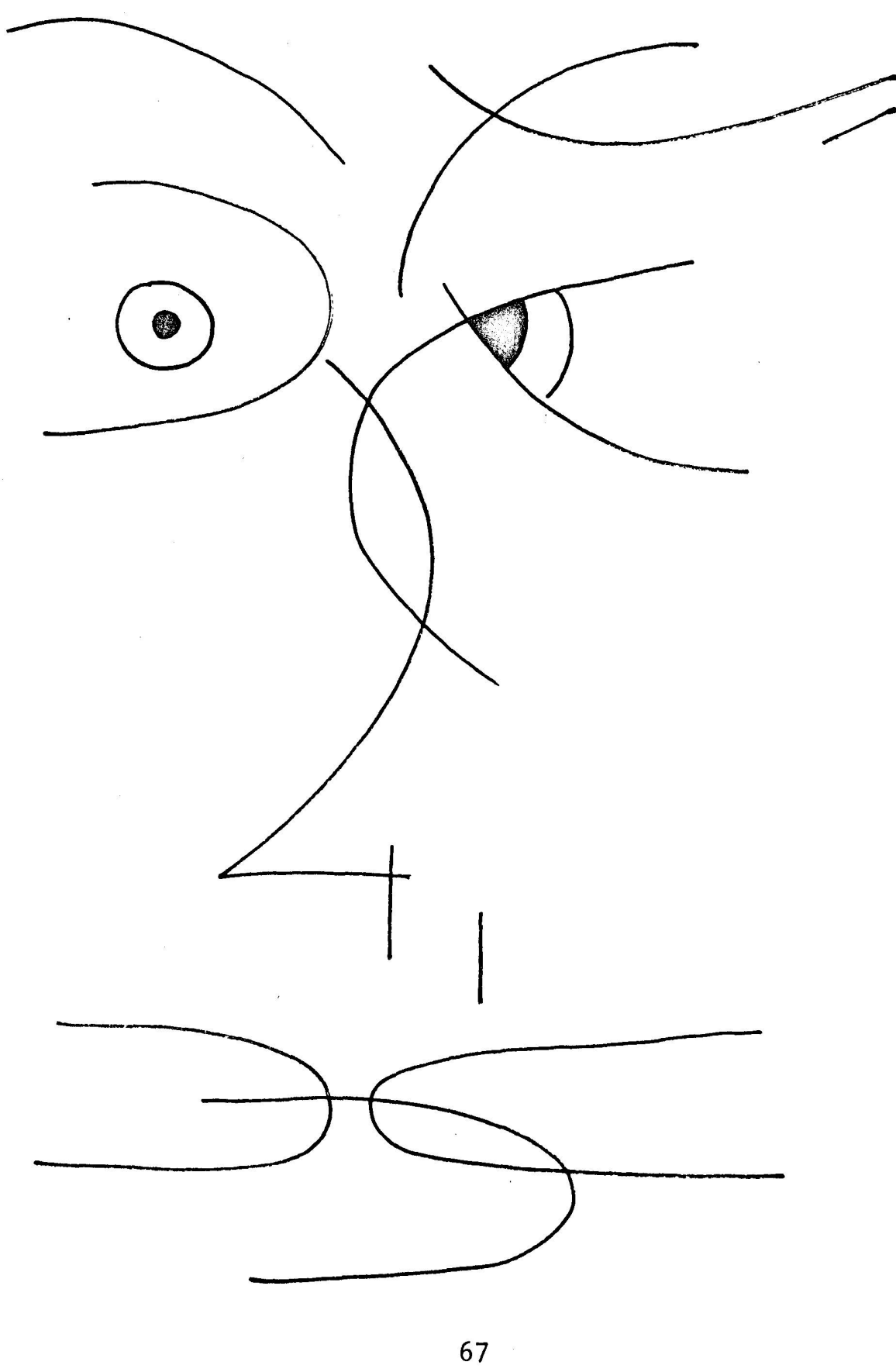

